\title{
Cryo-Electron Microscopy and Tomography Reveal Stepwise Expansion of the Bacteriophage $\phi 6$ Procapsid
}

D. Nemecek, ${ }^{*}$ J.B. Heymann, ${ }^{*}$ N. Cheng, ${ }^{*}$ J. Qiao, ${ }^{* *}$ L. Mindich, ${ }^{* *}$ and A.C. Steven*

* National Institute of Arthritis and Musculoskeletal and Skin Diseases, National Institutes of Health, 50 South Dr, Bethesda, MD 20892

** Department of Microbiology, Public Health Research Institute Center, University of Medicine and Dentistry of New Jersey, 225 Warren Street, Newark, NJ 07103

The double-stranded RNA bacteriophage $\phi 6$ that infects Pseudomonas syringae exhibits similarities to eukaryotic viruses of the Reoviridae and has been useful in studying simple RNA polymerases and packaging motors [1]. The inner shell of the triple-layered virion matures from an icosahedral precursor (procapsid) with recessed 5-fold vertices and a small internal volume. The three genomic segments are packaged in a specific order, and the current model is that this order is determined by the expansion state of the procapsid. We induced empty procapsids to expand by heating (Fig. 1 B), increasing salt concentration (Fig. 1 C) or acidification (Fig. 1 D), as well as intermediates obtained from RNA-packaged procapsids at higher salt concentrations (Figs. 1 F-H).

Samples were plunge-frozen and imaged on CM200 and Tecnai 12 (FEI) microscopes operating at $120 \mathrm{keV}$ for single particle analysis and tomography, respectively. Particles were initially aligned to a previous reconstruction of the procapsid [2] and the orientations iteratively refined using Bsoft [3]. In cases where more than one conformation were suspected, the maps were refined by mutually exclusive multi-reference alignments, yielding up to $\sim 18 \AA$ resolution reconstructions (Fig. 1). Tomograms of the heat-expanded procapsids were reconstructed and extracted particles aligned to standard icosahedral orientation using a missing wedge mask (Fig. 2). Extracted vertices from aligned procapsids were further classified using multi-reference alignment.

At room temperature, two intermediate states were identified in the sample of packaged procapsid exposed to high salt ( $>200 \mathrm{mM}$ ) (Figs. $1 \mathrm{G}, \mathrm{H}$ ), the more expanded one sometimes containing residual RNA (Fig. 1F). All of the treatments leading to expansion of the empty procapsid yielded a particle with apparently the same extent of expansion as in Fig. 1H (Figs. 1B-D). Examination of individual vertices of initial procapsids and the expanded intermediate at $60^{\circ} \mathrm{C}$ showed that they are flexible and oscillate between two states in each case (Figs. 2B,C). For the expanded intermediate, these two states of the vertices occur in almost every particle and correspond to the two intermediates seen at room temperature (Figs. 1H,G). The picture that emerges is that of fluctuations in the 5-fold vertices of the initial procapsid induced by environmental conditions, leading to an expansion event with similar fluctuations in a more expanded state. How these expansion intermediates relate to the sequential packaging of the genomic segments will be examined in future studies [4].

References

[1] L. Mindich, Virus Research 101 (2004) 83.

[2] A. Sen et al., J. Biol. Chem. 283 (2008) 12227.

[3] J.B. Heymann et al., J. Struct. Biol. 161 (2008) 232.

[4] This research was supported by the Intramural Research Program of NIAMS/NIH and by grant GM34352 to L.M. from the NIH. 

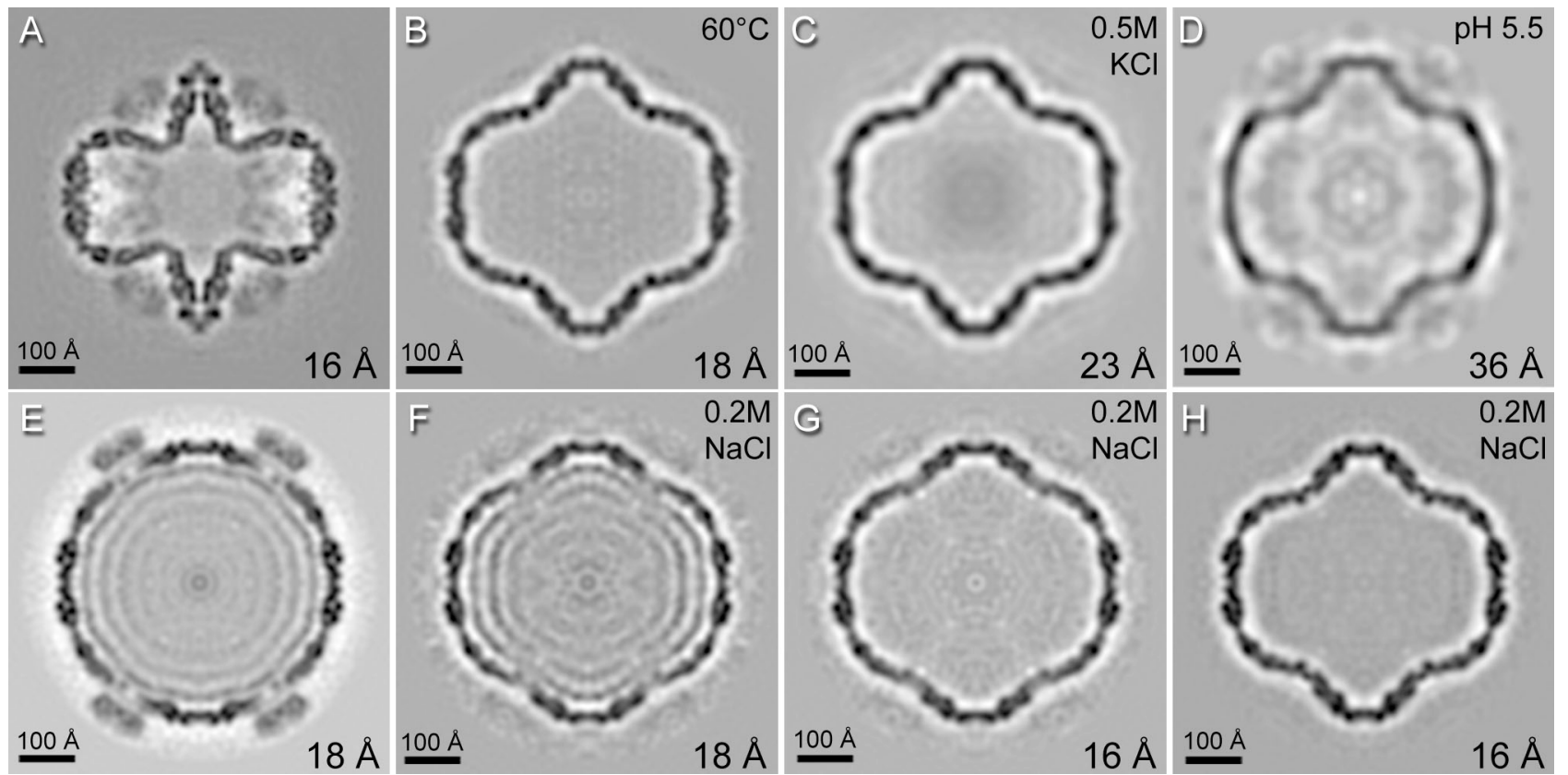

$100 \AA$

$18 \AA 100 \AA$

$18 \AA 100 \AA$

$16 \AA 100 \AA$

$16 \AA$

FIG. 1. Central sections through cryo-EM reconstructions of the initial $\phi 6$ procapsid (A) and its expansion intermediates obtained by heating (B), adding $\mathrm{KCl}(\mathrm{C})$ and acidification (D). Central sections through reconstructions of the RNA-packaged procapsid (E) and three species obtained at higher salt concentration (F-H). The extent of expansion in the intermediates in (B-D, H) are highly similar.
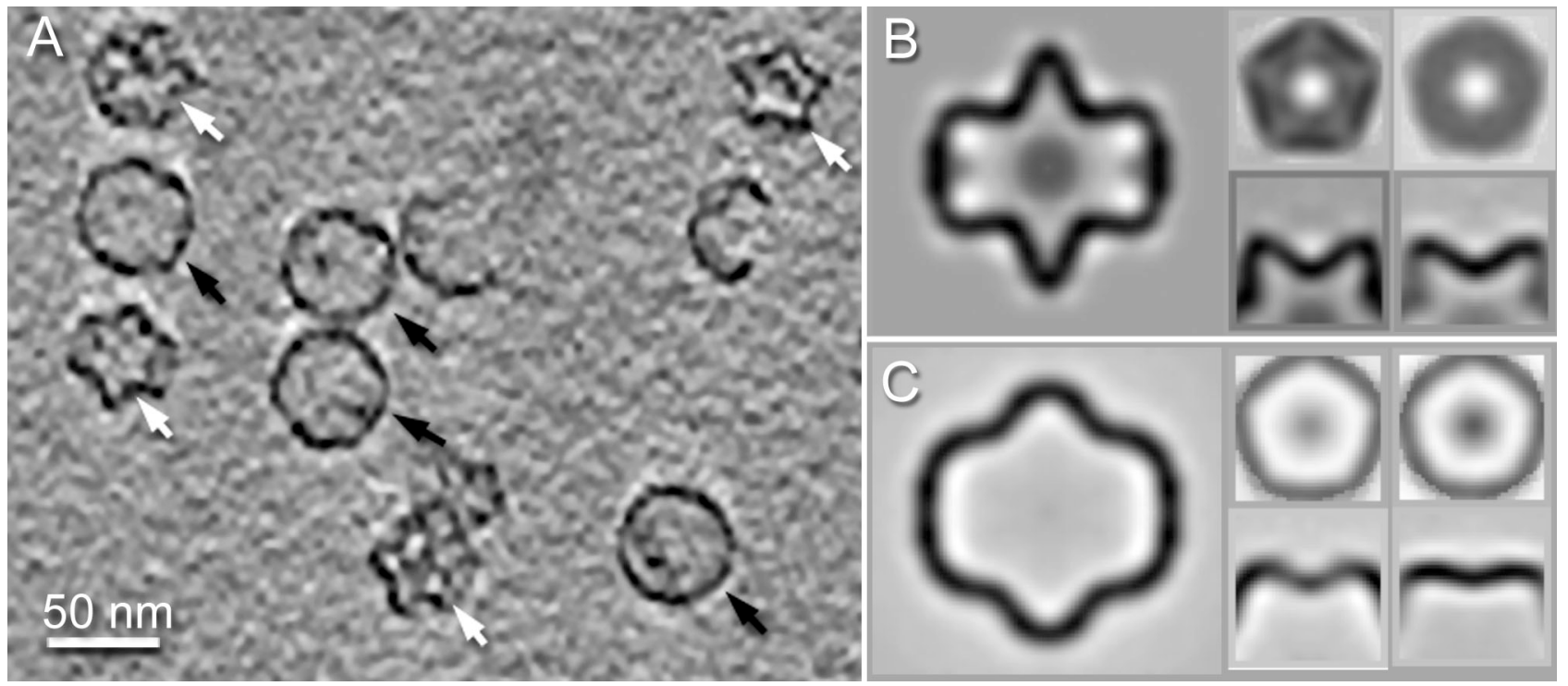

FIG. 2. (A) Section through a tomogram with initial (white arrows) and expanded (black arrows) procapsids. (B) Icosahedral average of aligned initial procapsids (left) and two class averages of extracted vertices viewed along (top) and perpendicular (bottom) to the 5-fold axis (right). (C) Icosahedral average of the expanded procapsid and two class averages of extracted vertices as in (B). 